\title{
MODELING OF NATURAL CONVECTION IN ELECTRONIC ENCLOSURES
}

\author{
Peter M. Teertstra, M. Michael Yovanovich and J. Richard Culham \\ Microelectronics Heat Transfer Laboratory \\ Department of Mechanical Engineering \\ University of Waterloo \\ Waterloo, Ontario, Canada N2L 3G1 \\ Phone: (519) 888-4586 \\ FAX: (519) 746-9141 \\ Email:pmt@mhtlab.uwaterloo.ca
}

\begin{abstract}
An analytical model is developed for natural convection from a single circuit board in a sealed electronic equipment enclosure. The circuit card is modelled as a vertical isothermal plate located at the center of an isothermal, cuboid shaped enclosure. A composite model is developed based on asymptotic solutions for three limiting cases: pure conduction, laminar boundary layer convection and transition flow convection. The conduction shape factor and natural convection models are validated using data from CFD simulations for a wide range of enclosure geometries and flow conditions. The model is shown to be in good agreement, within $10 \%$ RMS, with the numerical data for all test configurations.
\end{abstract}

\begin{tabular}{ll} 
& \multicolumn{1}{c}{ NOMENCLATURE } \\
$A$ & area, $m^{2}$ \\
$b$ & plate to enclosure spacing, $m$ \\
$c_{p}$ & specific heat, $J / k g K$ \\
$d_{\text {eff }}$ & effective diameter, $m$ \\
$F(P r)$ & Prandtl number function \\
$g$ & gravitational acceleration, $m / s^{2}$ \\
$G_{\mathcal{L}}$ & body gravity function \\
$k$ & thermal conductivity, $W / m K$ \\
$L$ & length, $m$ \\
$\mathcal{L}$ & general scale length, $m$ \\
$n, m$ & combination parameters \\
$N u_{\mathcal{L}}$ & Nusselt number, $\equiv Q \mathcal{L} / k A_{i}\left(T_{i}-T_{o}\right)$ \\
$P r$ & Prandtl number, $\equiv \mathrm{v} / \alpha$ \\
$Q$ & total heat transfer rate, $W$ \\
$R$ & thermal resistance, $\equiv \Delta T / Q, K / W$ \\
$R a_{\mathcal{L}}$ & Rayleigh number, $\equiv g \beta \Delta T \mathcal{L}^{3} / \mathrm{v \alpha}$ \\
$S$ & conduction shape factor, $\equiv 1 / R k, 1 / m$ \\
$S_{\mathcal{L}}^{\star}$ & dimensionless shape factor, $\equiv S \mathcal{L} / A_{i}$ \\
$T_{f}$ & film temperature, $\equiv\left(T_{i}+T_{o}\right) / 2,{ }^{o} C$ \\
$T_{i}$ & plate temperature, ${ }^{o} C$ \\
$T_{o}$ & enclosure wall temperature, ${ }^{o} C$ \\
$V$ & volume, $m^{3}$ \\
$W$ & width, $m$ \\
& \\
Greek & Symbols \\
$\alpha$ & thermal diffusivity, $m^{2} / s$ \\
$\beta$ & thermal expansion, $1 / K$ \\
$\delta x_{m i n}$ & minimum control volume thickness, $m$ \\
$\Delta T$ & temperature difference, $\equiv T_{i}-T_{o},{ }^{o} C$
\end{tabular}

\begin{tabular}{ll}
$\mu$ & dynamic viscosity, $\mathrm{Ns} / \mathrm{m}^{2}$ \\
$\nu$ & kinematic viscosity, $\mathrm{m}^{2} / \mathrm{s}$ \\
$\rho$ & mass density, $\mathrm{kg} / \mathrm{m}^{3}$ \\
\multicolumn{2}{l}{ Subscripts } \\
$1 / 8$ & one-eighth symmetry results \\
$1 / 4$ & one-quarter symmetry results \\
bl & boundary layer flow asymptote \\
eff & effective \\
$f$ & fluid \\
$L B$ & lower bound \\
$U B$ & upper bound \\
tr & transition flow asymptote \\
$i$ & inner \\
$o$ & outer
\end{tabular}

\section{INTRODUCTION}

Natural convection heat transfer in the cavity formed between a heated vertical plate and its surrounding cooled cuboid-shaped enclosure is of some interest in the design of sealed electronic equipment for harsh environments and outdoor applications. Many of these applications rely on convection in the enclosed air space to remove heat from the circuit board and components and transfer it to the enclosure, where it is dissipated by conduction through the walls and convection from the exterior to the surrounding environment.

Current practice for the design of sealed enclosures for electronic equipment usually involves extensive use of numerical CFD simulations and experimental testing of prototypes and mock-ups. The time consuming and costly nature of these methods make them poor choices for activities such as parametric analysis or "what-if" studies, precluding their usefulness during the preliminary phases of product design. Quick, easy to use modelling procedures for natural convection in these enclosure systems represent an effective alternative to prototype testing or CFD simulations. Analytically based models that utilize readily available information, such as physical dimensions, thermophysical properties and average temperature or heat flux values for boundary conditions, provide the engineer with a set of analysis tools perfectly suited for the initial phases of the design. These models can also be easily implemented into design tools using a variety of platforms, including symbolic mathematics software such as Maple or MathCad, or in a spreadsheet environment such as Excel. The use of these analytically based 
design tools will speed up the design process, reduce costs and result in the development of more reliable and cost effective equipment.

The literature contains many publications examining the topic of natural convection from a heated internal body to its surrounding cooled enclosure for a variety of inner body and outer boundary shape and boundary condition combinations. Of specific interest for sealed equipment enclosures is the geometry formed between the vertical flat plate and a cuboid-shaped enclosure. A number of studies are presented in the literature for the vertical flat plate with protruding discrete heat sources in a sealed enclosure, such as Park [1] and Tang and Yoshi [2]. Other publications present experimental or CFD simulation results for arrays of two or more parallel vertical plates, such as Symons et al. [3]. Yang and Tao [4] present a combined numerical and experimental study of three dimensional natural convection in a cubical enclosure with an internally isolated, heated vertical plate. These data are limited to a single cubical enclosure / rectangular plate geometry, and the correlation provided by the authors for the average Nusselt is limited both in geometry and in the range of Rayleigh number over which it is applicable.

The objectives of the current study are to develop and validate an analytically based model for laminar natural convection from an isolated, vertical isothermal flat plate to an isothermal, cuboid-shaped enclosure. The model will be valid for the full range of Rayleigh number, from laminar boundary layer convection in large scale enclosures to conduction dominated heat transfer in small or micro-scale devices. This model will be validated using data from numerical CFD simulations for a variety of enclosure geometries and flow conditions.

\section{PROBLEM DESCRIPTION}

The problem of interest involves natural convection from an isothermal, vertical rectangular plate to its surrounding isothermal cuboid-shaped enclosure. A schematic of the relevant dimensions is presented in Fig. 1. The vertical plate is located at the center of the enclosure, and isothermal boundary conditions $T_{i}$ and $T_{o}$ are imposed on the plate and enclosure walls, respectively. All dimensions of the plate and enclosure are specified in relation to the length of the plate, $L_{i}$, using the following dimensionless parameters:

$$
\frac{L_{o}}{L_{i}}, \quad \frac{L_{o}}{W_{o}}, \quad \frac{L_{i}}{W_{i}}, \quad \frac{b}{L_{o}}
$$

The independent parameters are non-dimensionalized by the Rayleigh number, defined using the overall temperature difference, $\Delta T=T_{i}-T_{o}$, and the square root of the surface area of the vertical plate as the scale length:

$$
R a_{\sqrt{A_{i}}}=\frac{g \beta \Delta T\left(\sqrt{A_{i}}\right)^{3}}{\nu \alpha}, \quad A_{i}=2 L_{i} W_{i}
$$

where the thermophysical properties are determined at the fluid temperature, which can be approximated by the arithmetic mean:

$$
T_{f}=\frac{T_{i}+T_{o}}{2}
$$

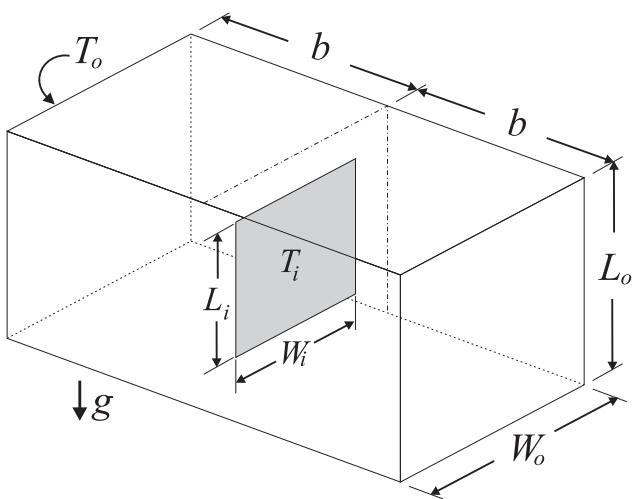

Fig. 1 Schematic of Enclosure Geometry

The quantity of interest in the study, the total heat transfer rate $Q$ between the plate and the enclosed through the fluid region is non-dimensionalized by the Nusselt number defined using the same temperature difference and length scale as $R a_{\sqrt{A_{i}}}$ :

$$
N u_{\sqrt{A_{i}}}=\frac{Q}{k \sqrt{A_{i}} \Delta T}
$$

For the limiting case of small Rayleigh number, the heat transfer through the enclosure becomes conduction dominated and the Nusselt number is equivalent to the conduction shape factor:

$$
S_{\sqrt{A_{i}}}^{\star}=\frac{Q}{k \sqrt{A_{i}} \Delta T}=\frac{S}{\sqrt{A_{i}}} \text { where } S=\frac{1}{R k}
$$

\section{MODEL DEVELOPMENT}

Teertstra [5] and Teertstra et al. [6] present an analytical method for calculating the total heat transfer rate in regions bounded by an arbitrarily shaped heated inner body and an arbitrarily shaped surrounding cooled enclosure. The general formulation of the model represents a combination of three asymptotic terms, corresponding to the three modes / methods of heat transfer present in the enclosed fluid region:

$$
N u_{\sqrt{A_{i}}}=S_{\sqrt{A_{i}}}^{\star}+\frac{1}{\left[\left(\frac{1}{N u_{\mathrm{tr}}}\right)^{n}+\left(\frac{1}{N u_{\mathrm{bl}}}\right)^{n}\right]^{1 / n}}
$$

where $S_{\sqrt{A_{i}}}^{\star}$, the conduction shape factor, is combined with the remaining convective terms using linear superposition. The convective terms, $N u_{\mathrm{bl}}$, laminar boundary layer flow, and $N u_{\mathrm{tr}}$, transition flow at low Rayleigh number, are combined using the Churchill and Usagi [7] composite solution method. $N u_{b l}$ represents the limiting case of high Rayleigh number heat transfer by laminar natural convection at the inner and outer walls, where the fluid in the core region is quiescent and of uniform temperature. The other convective limit, $N u_{\text {tr }}$, corresponds to the limiting case of low Rayleigh number, where boundary layers at the heated and cooled walls grow quickly, merge and overlap. As a result, the temperature profile approaches that of pure conduction, fluid movement is induced in the core region, and convective heat transfer occurs at the top and bottom regions of the enclosure. The three asymptotic solutions as well as the resulting three term model is presented in Fig. 2. The following 


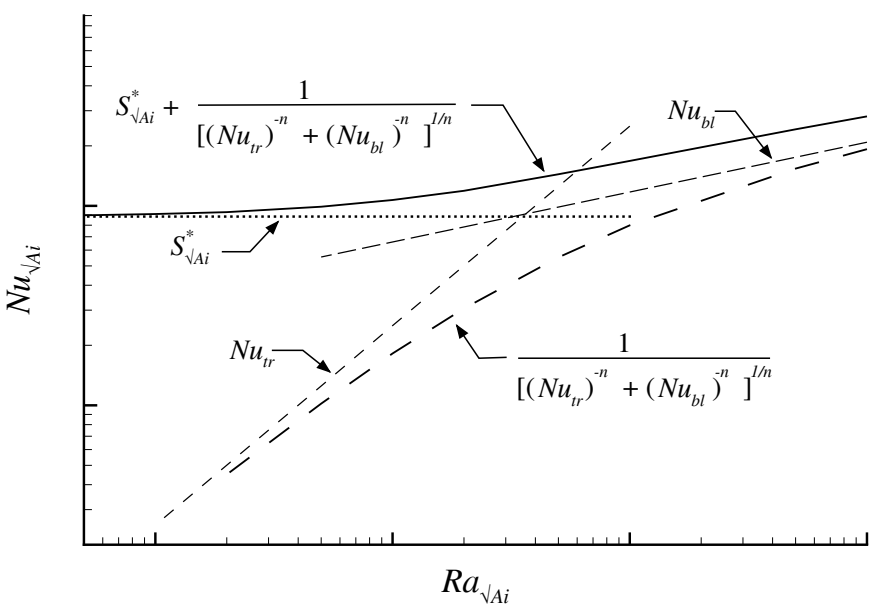

Fig. 2 Composite Model for Enclosure Convection

sections describe in detail the development of each of the three asymptotic solutions to these limiting cases.

\section{Conduction Shape Factor}

The dimensionless conduction shape factor is modeled using the Churchill and Usagi [7] composite technique, which combines asymptotic solutions for small and large values of the independent parameter, the gap spacing between the vertical plate and the opposite enclosure wall, $b / L_{o}$.

$$
S_{\sqrt{A_{i}}}^{\star}=\left[\left(S_{b / L_{o} \rightarrow 0}^{\star}\right)^{m}+\left(S_{b / L_{o} \rightarrow \infty}^{\star}\right)^{m}\right]^{1 / m}
$$

The two limiting cases corresponding to small and large values of the dimensionless gap thickness, $b / L_{o}$, are combined as shown in Fig. 3.

The first asymptotic solution, $b / L_{o} \rightarrow 0$, is modelled based on the limiting case of one-dimensional conduction in the gap. This simple conduction relationship is expressed as a conduction shape factor by:

$$
S=\frac{1}{k R}=\frac{1}{k} \frac{1}{\left(\frac{b}{k A_{i}}\right)}=\frac{A_{i}}{b}
$$

This conduction shape factor is non-dimensionalized by the scale length $\sqrt{A_{i}}$ to give the following asymptotic solution:

$$
S_{b / L_{o} \rightarrow 0}^{\star}=\frac{S}{\sqrt{A_{i}}}=\frac{\sqrt{A_{i}}}{b}
$$

Re-casting this expression in terms of the dimensionless geometric parameters from Eq. (1) yields:

$$
S_{\sqrt{A_{i}}}^{\star}=\frac{\sqrt{2}}{\left(\frac{b}{L_{o}}\right)\left(\frac{L_{o}}{L_{i}}\right) \sqrt{\frac{L_{i}}{W_{i}}}}
$$

The remaining asymptotic solution corresponds to the limiting case of large gap spacing, where the conduction shape factor becomes independent of $b / L_{o}$. For this limit the enclosed region

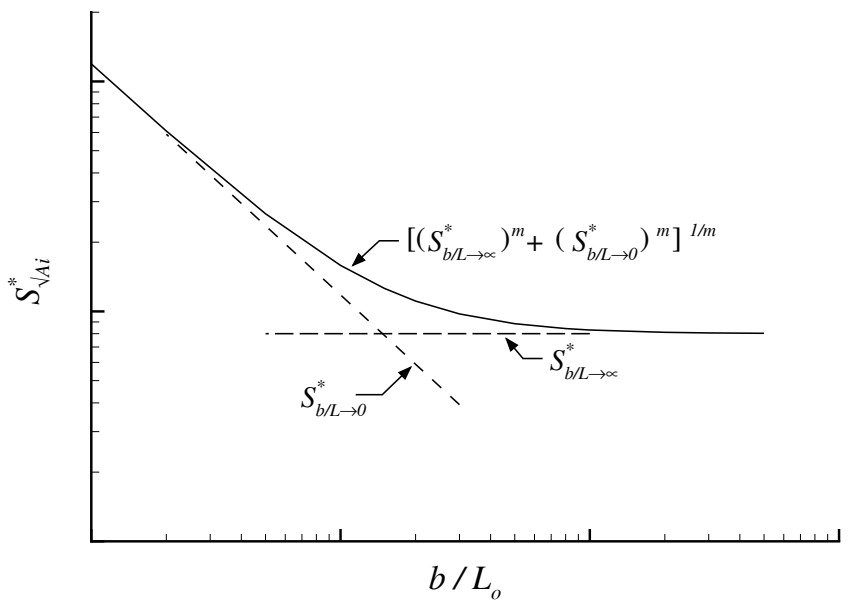

Fig. 3 Composite Model for Conduction Shape Factor

is modelled using an equivalent thermal resistance network consisting of a series combination of resistances for the plate and an equivalent spherical surface representing the cuboid shaped enclosure boundary:

$$
R=R_{\text {plate }}-R_{\text {sphere }}
$$

where $R_{\text {plate }}$ is the conductive resistance of an isothermal plate in a full space region and $R_{\text {sphere }}$ is the conductive resistance for the equivalent sphere in a full space. The conductive resistance for the plate is determined based on the dimensionless conduction shape factor expression presented by Yovanovich [8]:

$$
\begin{aligned}
R_{\text {plate }} & =\frac{1}{S k}=\frac{1}{k \sqrt{A_{i}}} \frac{1}{S_{\text {plate }}^{\star}} \\
S_{\text {plate }}^{\star} & =\frac{0.8\left(1+\sqrt{L_{i} / W_{i}}\right)^{2}}{\sqrt{L_{i} / W_{i}}} \\
R_{\text {plate }} & =\frac{1.25}{\sqrt{2} k W_{i}} \frac{1}{\left(1+\sqrt{L_{i} / W_{i}}\right)^{2}}
\end{aligned}
$$

$R_{\text {sphere }}$ is determined based on the available analytical solution for the dimensionless conduction shape factor of a sphere in a full space region [8]:

$$
\begin{aligned}
R_{\text {sphere }} & =\frac{1}{S k}=\frac{1}{k \sqrt{A_{i}}} \frac{1}{S_{\text {sphere }}^{\star}} \\
S_{\text {sphere }}^{\star} & =2 \sqrt{\pi}
\end{aligned}
$$

The equivalent sphere is related to the geometry of the enclosure through an effective diameter, defined based on the arithmetic mean of the length and width dimensions:

$$
d_{\mathrm{eff}}=\frac{L_{o}+W_{o}}{2}
$$

The resulting expression for the conductive resistance for the equivalent sphere becomes:

$$
R_{\text {sphere }}=\frac{1}{k \pi W_{o}\left(L_{o} / W_{o}+1\right)}
$$


Combining the two resistance expressions into Eq. (11) and recasting in terms of the dimensionless conduction shape factor yields:

$$
S_{b / L_{o} \rightarrow \infty}^{\star}=\frac{\sqrt{W_{i} / L_{i}}}{\left[\frac{1.25}{\left(1+\sqrt{L_{i} / W_{i}}\right)^{2}}-\frac{\sqrt{2}\left(W_{o} / W_{i}\right)}{\pi\left(L_{o} / W_{o}+1\right)}\right]}
$$

Using the composite method to combine the asymptotic solutions for small and large $b / L_{o}$, Eqs. (10) and (15) gives the following expression for the conduction shape factor for the enclosure geometry:

$$
\begin{aligned}
S_{\sqrt{A_{i}}}^{\star} & =\left[\left(\frac{\sqrt{2}}{\left(b / L_{o}\right)\left(L_{o} / L_{i}\right) \sqrt{L_{i} / W_{i}}}\right)^{m}\right. \\
& \left.+\left(\sqrt{\frac{W_{i}}{L_{i}}}\left[\frac{1.25}{\left(1+\sqrt{\frac{L_{i}}{W_{i}}}\right)^{2}}-\frac{\sqrt{2}\left(W_{o} / W_{i}\right)}{\pi\left(\frac{L_{o}}{W_{o}}+1\right)}\right]^{-1}\right)^{m}\right]^{1 / m}
\end{aligned}
$$

where the combination parameter $m$ is determined based on a comparison with numerical data for a wide variety of enclosures, as demonstrated in a subsequent part of the study.

\section{Laminar Boundary Layer Convection}

The first of the two asymptotic solutions for convection in the enclosed fluid region represents the limiting case of high Rayleigh number, laminar boundary layer flows over the vertical flat plate and the enclosure walls. At this limit it is assumed that the fluid in the core region is of uniform temperature. For this asymptote Teertstra [5] presents the following analytical model for the Nusselt number based on a combination of laminar boundary layer models on the inner (heated) and outer (cooled) surfaces:

$$
N u_{\mathrm{bl}}=\frac{F(\operatorname{Pr}) G_{\sqrt{A_{i}}} \operatorname{Ra} \sqrt{\sqrt{A_{i}}}}{\left[1+\left(\frac{A_{i}}{A_{o}}\right)^{7 / 10}\left(\frac{G_{\sqrt{A_{i}}}}{G_{\sqrt{A_{o}}}}\right)^{4 / 5}\right]^{5 / 4}}
$$

where the Prandtl number function, $F(P r)$, as defined by Churchill and Churchill [9]:

$$
F(P r)=\frac{0.67}{\left[1+\left(\frac{0.5}{P r}\right)^{9 / 16}\right]^{4 / 9}}
$$

evaluates to $F(P r)=0.513$ for air at STP $(P r=0.7)$.

The body gravity functions for the inner and outer boundaries, $G_{\sqrt{A_{i}}}$ and $G_{\sqrt{A_{o}}}$, are calculated based on the methods described by Lee et al. [11]. For the vertical plate, the body gravity function is determined by:

$$
G_{\sqrt{A_{i}}}=2^{1 / 8}\left(\frac{W_{i}}{L_{i}}\right)^{1 / 8}
$$

The body gravity function for the outer cuboid shaped boundary is analyzed based on a model presented by Jafarpur and Yovanovich [10]

$$
G_{\sqrt{A_{o}}}=2^{1 / 8}\left[\frac{0.625(2 b)^{4 / 3} W_{o}+L_{o}\left(2 b+W_{o}\right)^{4 / 3}}{\left[L_{o} W_{o}+2 b\left(W_{o}+L_{o}\right)\right]^{7 / 6}}\right]^{3 / 4}
$$

where $2 b \geq W_{o}$. When the gap spacing of the enclosure is reduced and $2 b<W_{o}$, the parameters $2 b$ and $W_{o}$ are interchanged in Eq. (20). Re-casting the body gravity function expression in terms of the dimensionless parameters defined in Eq. (1) yields:

$$
G_{\sqrt{A_{o}}}=2^{1 / 8}\left[\frac{0.625\left(\frac{2 b}{L_{o}} \frac{L_{o}}{W_{o}}\right)^{4 / 3}+\frac{L_{o}}{W_{o}}\left(\frac{2 b}{L_{o}}+\frac{L_{o}}{W_{o}}\right)^{4 / 3}}{\left[\frac{L_{o}}{W_{o}}+\frac{2 b}{L_{o}} \frac{L_{o}}{W_{o}}\left(\frac{L_{o}}{W_{o}}+1\right)\right]^{7 / 6}}\right]^{3 / 4}
$$

for $2 b \geq W_{o}$ and

$$
G_{\sqrt{A_{o}}}=2^{1 / 8}\left[\frac{0.625\left(\frac{W_{o}}{L_{o}} \frac{L_{o}}{2 b}\right)^{4 / 3}+\frac{L_{o}}{2 b}\left(\frac{W_{o}}{L_{o}}+\frac{L_{o}}{2 b}\right)^{4 / 3}}{\left[\frac{L_{o}}{2 b}+\frac{W_{o}}{L_{o}} \frac{L_{o}}{2 b}\left(\frac{L_{o}}{2 b}+1\right)\right]^{7 / 6}}\right]^{3 / 4}
$$

when $2 b<W_{o}$. The ratio of the inner to outer surface areas, $A_{i} / A_{o}$ is determined based on:

$$
\frac{A_{i}}{A_{o}}=\frac{L_{o} / W_{o}}{\left(\frac{L_{o}}{L_{i}}\right)^{2}\left(\frac{L_{i}}{W_{i}}\right)\left[1+\frac{2 b}{L_{o}}\left(\frac{L_{o}}{W_{o}}+1\right)\right]}
$$

For the example of a square plate, $L_{i} / W_{i}=1$, inside an enclosure with a square cross section, $L_{o} / W_{o}=1$, relative size $L_{o} / L_{i}=1.2$ and gap spacing $b / L_{o}=1$, the laminar boundary layer convection asymptote can be determined as follows:

$$
\begin{aligned}
G_{\sqrt{A_{i}}} & =2^{1 / 8} \approx 1.091 \\
G_{\sqrt{A_{o}}} & =2^{1 / 8}\left[\frac{0.625(2)^{4 / 3}+(3)^{4 / 3}}{(1+2(2))^{7 / 6}}\right] \approx 1.01 \\
\frac{A_{i}}{A_{o}} & =\frac{1}{(1.2)^{2}[1+2(2)]} \approx 0.139 \\
N u_{\mathrm{bl}} & =\frac{(0.513)(1.091)}{\left[1+(0.139)^{7 / 10}(1.091 / 1.01)^{4 / 5}\right]^{5 / 4}} \cdot R a_{\sqrt{A_{i}}}^{1 / 4}
\end{aligned}
$$

For the limiting case of a large enclosure, $A_{i} / A_{o} \rightarrow 0, N u_{\mathrm{bl}}$ reduces to the analytical solution for the vertical flat plate in a full space region.

\section{Transition Flow Convection}

The remaining asymptotic solution for convection in the enclosure corresponds to the limiting case of low Rayleigh number flow, where the boundary layers have merged and a near-linear 
temperature distribution is established in the core region. In this case, convective heat transfer occurs within the small recirculation regions that are established at the top and bottom of the enclosure. A model for this transition flow limit is presented by Teertstra [5] based on the analytical solution of Batchelor [12] for the tall vertical cavity formed between differentially heated parallel plates:

$$
N u_{\delta}=\frac{1}{720} \frac{\delta}{L} R a_{\delta}
$$

where $\delta$ is the gap spacing between the plates and $L$ is the height of the cavity. Teertstra [5] extended this expression to include enclosures formed between arbitrarily shaped boundaries, including the current geometry. Based on a simplification of this general model, the following expression for the transition flow asymptote is proposed:

$N u_{\mathrm{tr}}=\frac{\sqrt{2}}{360} \frac{\sqrt{W_{i} / L_{i}}}{\left[\left(1+L_{o, U B} / L_{i}\right)\left(1+L_{o, L B} / L_{i}\right)\right]^{1 / 2}}\left(\frac{\delta_{\mathrm{eff}}}{\sqrt{A_{i}}}\right)^{3} R a_{\sqrt{A_{i}}}$

where $\delta_{\text {eff }} / \sqrt{A_{i}}$ represents the dimensionless effective gap spacing and the term in the denominator is a mean of the inner and outer boundary lengths.

The effective gap spacing is determined for an equivalent spherical cavity, where both the surface area of the inner body as well as the enclosed volume are preserved, resulting in the following expression:

$$
\begin{aligned}
\frac{\delta_{\text {eff }}}{\sqrt{A_{i}}} & =\frac{1}{2 \sqrt{\pi}}\left[\left(6 \sqrt{\pi} \frac{V}{A_{i}^{3 / 2}}+1\right)^{1 / 3}-1\right] \\
& =\frac{1}{2 \sqrt{\pi}}\left[\left(3 \sqrt{2 \pi}\left(\frac{L_{o}}{L_{i}}\right)^{3}\left(\frac{L_{i}}{W_{i}}\right)^{3 / 2}\left(\frac{W_{o}}{L_{o}}\right)\left(\frac{b}{L_{o}}\right)+1\right)^{1 / 3}-1\right]
\end{aligned}
$$

The upper and lower bounds for the outer boundary length, $L_{o, U B}$ and $L_{o, L B}$, are determined based on the minimum and maximum lengths between the stagnation points along the outer wall of the enclosure:

$$
L_{o, U B} \approx L_{o}, \quad L_{o, L B}=2 b+L_{o}
$$

The resulting expression for the transition flow asymptote is:

$$
N u_{\mathrm{tr}}=\frac{\sqrt{2}}{360} \frac{\sqrt{W_{i} / L_{i}}\left(\delta_{\mathrm{eff}} / \sqrt{A_{i}}\right)^{3}}{\left[\left(1+\frac{L_{o}}{L_{i}}\right)\left(1+\frac{L_{o}}{L_{i}}\left(\frac{2 b}{L_{o}}+1\right)\right)\right]^{1 / 2}} R a_{\sqrt{A_{i}}}
$$

where $\delta_{\text {eff }} / \sqrt{A_{i}}$ is calculated using Eq. (26).

For the test case examined in the last section, $L_{i} / W_{i}=$ $L_{o} / W_{o}=1, L_{o} / L_{i}=1.2$ and $b / L_{o}=1$, the transition flow asymptote can be determined as a function of Rayleigh number as follows:

$$
\begin{aligned}
\frac{\delta_{\mathrm{eff}}}{\sqrt{A_{i}}} & =\frac{1}{2 \sqrt{\pi}}\left[\left(3 \sqrt{2 \pi}(1.2)^{3}+1\right)^{1 / 3}-1\right] \approx 0.398 \\
N u_{\mathrm{tr}} & =\frac{(\sqrt{2} / 360)(0.398)^{3}}{[(1+1.2)(1+1.2(3))]^{1 / 2}} \cdot R a_{\sqrt{A_{i}}}
\end{aligned}
$$

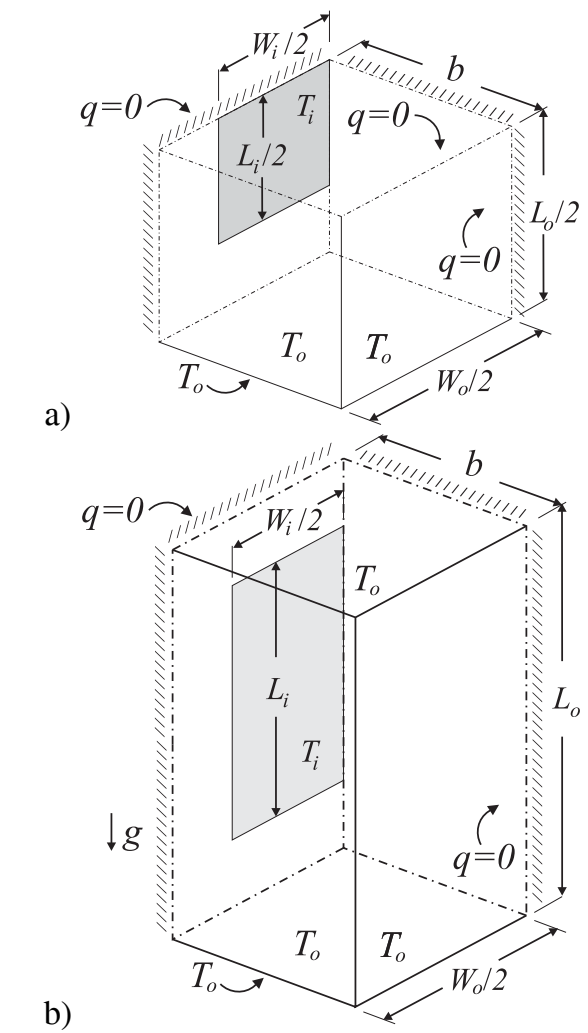

Fig. 4 CFD Model Solution Domains: a) Flotherm [13]

Conduction Model; b) Icepak [14] Convection Model

\section{CFD SIMULATIONS}

To validate the analytical models for conduction shape factor and natural convection, CFD simulations were performed for a wide range of enclosure geometries and flow conditions using two different commercial computational fluid dynamics (CFD) packages, Flotherm [13] and Icepak [14]. In the following section the details of the numerical simulations for both the conduction and convection solutions will be presented.

\section{Conduction Shape Factor}

The conduction shape factor model will be validated using data from CFD simulations performed using Flotherm [13], a commercial finite volume based software package. Conduction-only simulations were performed for the $1 / 8$ symmetry geometry presented in Fig. 4 a) with constant thermophysical properties of air at $20^{\circ} \mathrm{C}$. An isothermal source, $T_{i}=40^{\circ} \mathrm{C}$, was located on one domain boundary, and isothermal boundary conditions, $T_{o}=20^{\circ} \mathrm{C}$, were imposed on the three remaining exterior (nonsymmetry) boundaries. The total heat transfer from the source, $Q_{1 / 8}$, predicted by the CFD simulations for the $1 / 8$ symmetry case was converted to an equivalent conduction shape factor by:

$$
S_{\sqrt{A_{i}}}^{\star}=\frac{Q_{1 / 8} \times 8}{k \sqrt{A_{i}} \Delta T}
$$

In order to ensure independence of the numerical results from the number or size of control volumes, a grid convergence study was performed for the following enclosure geometry:

$$
L_{i}=0.1 m, \quad L_{o} / L_{i}=1.2, \quad L_{i} / W_{i}=L_{o} / W_{o}=b / L_{o}=1
$$




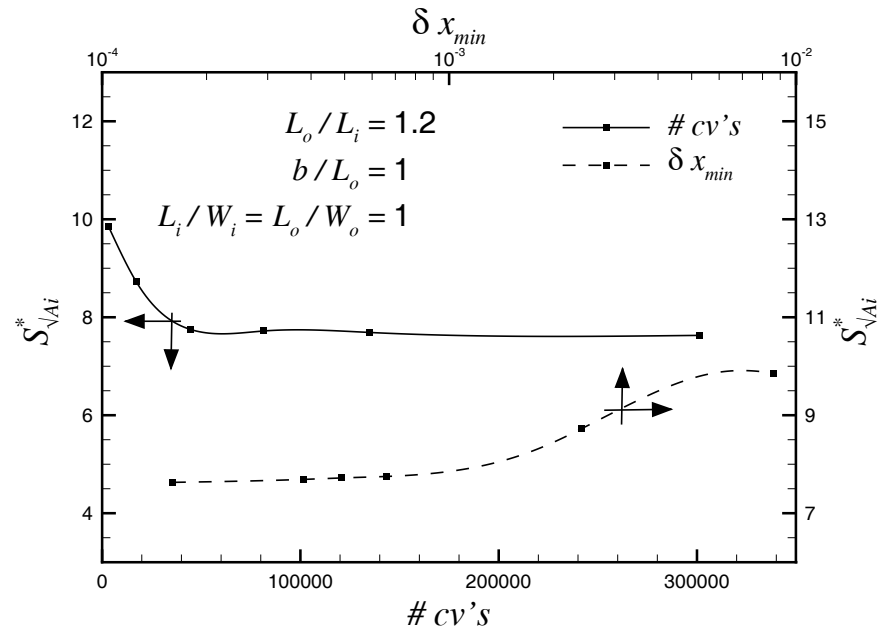

Fig. 5 Grid Convergence: Conduction Simulations

In Fig. 5 the results of the grid convergence study are presented in two ways. The dimensionless conduction shape factor is plotted versus the total number of control volumes and the minimum control volume thickness in the $x$-direction, normal and adjacent to the heat source. Figure 5 clearly demonstrates that sufficient control volume refinement is possible such that grid independence can be achieved. All subsequent simulations will be performed using similar grid parameters.

Conduction simulations were performed using the Flotherm [13] package for a variety of enclosure geometry configurations, including the following:

$$
\begin{aligned}
L_{o} / L_{i} & =1.05,1.2,1.6,2.0 \\
L_{i} / W_{i} & =0.5,1,2 \\
L_{o} / W_{o} & =0.5,1,2 \\
b / L_{o} & =1,0.8,0.5,0.3,0.2,0.15,0.1,0.05
\end{aligned}
$$

The results of these numerical simulations will be compared with the analytical model predictions in the next section.

\section{Natural Convection}

The full model for natural convection in the enclosure will be validated with data from CFD simulations performed in Icepak [14], a finite element based commercial CFD package. Numerical simulations were performed assuming laminar flow and no radiation heat transfer in the $1 / 4$ symmetry solution domain shown in Fig. 4 b). An isothermal source, $T_{i}=40^{\circ} \mathrm{C}$, was located at the $x=0$ boundary and an isothermal condition, $T_{o}=20^{\circ} \mathrm{C}$, was imposed on the four remaining non-symmetry boundaries. The total heat transfer from the source $Q_{1 / 4}$ provided by the numerical simulation was non-dimensionalized by:

$$
N u_{\sqrt{A_{i}}}=\frac{Q_{1 / 4} \times 4}{k \sqrt{A_{i}} \Delta T}
$$

In order to simulate a wide range of Rayleigh number variation without changing the size of the solution domain, which would require changes to feature locations, grid parameters, etc., the density of the fluid in the enclosure was varied from its default

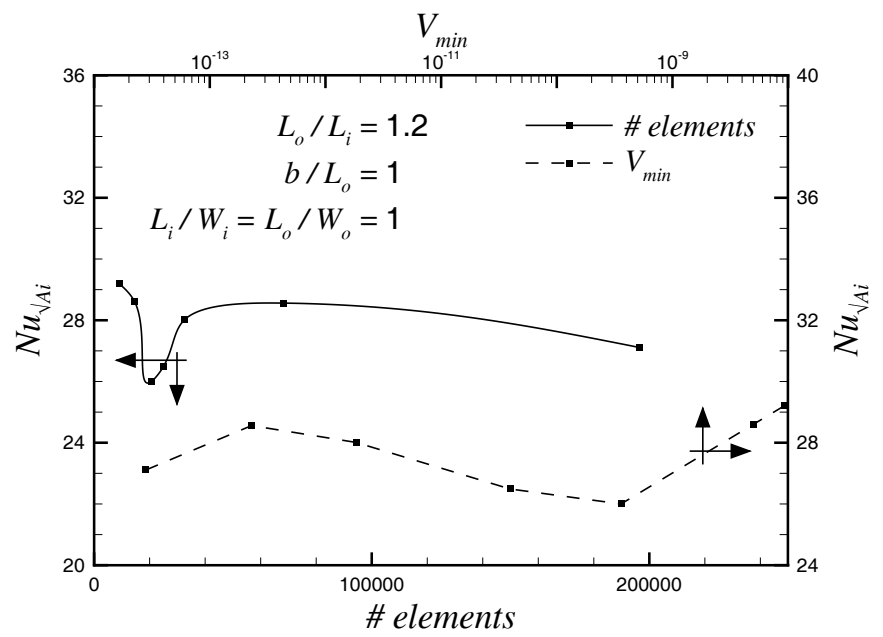

Fig. 6 Grid Convergence: Convection Simulations

value of $\rho=1.1614 \mathrm{~kg} / \mathrm{m}^{3}$ in 5 steps to $\rho=0.011614 \mathrm{~kg} / \mathrm{m}^{3}$. The corresponding values of the Rayleigh number are determined by re-casting $R a_{\sqrt{A_{i}}}$ in terms of $\rho$ :

$$
R a_{\sqrt{A_{i}}}=\frac{g \beta \Delta T\left(\sqrt{A_{i}}\right)^{3} c_{p} \rho^{2}}{\mu k}
$$

The range of Rayleigh number resulting from this variation in density is:

$$
5 \times 10^{2} \leq R a_{\sqrt{A_{i}}} \leq 5 \times 10^{6}
$$

All other thermophysical properties in the finite element simulations are treated as constant values for air at $T=20^{\circ} \mathrm{C}$.

As in the case of the conduction simulations, a grid convergence study was performed for a typical enclosure geometry:

$$
L_{i}=0.1 m, \quad L_{o} / L_{i}=1.2, \quad L_{i} / W_{i}=L_{o} / W_{o}=b / L_{o}=1
$$

The results of the grid convergence study are presented in Fig. 6 as a function of the total number of elements and the minimum volume of the elements located adjacent to the heat source. The convergence study indicates that a sufficient level of discretization has been achieved such that the results of the numerical simulations are independent of the grid. All subsequent CFD convection simulations used the same level of grid refinement.

The natural convection simulations performed in Icepak [14] utilize the same set of enclosure geometries as those used in the conduction modelling in Flotherm [13], as presented in Eq. (29). All results of the CFD convection simulations will be compared with the predictions of the analytical model in the following section.

\section{MODEL VALIDATION}

With the model development completed and the numerical CFD simulations providing data for the total heat transfer rate in the enclosure, the data are used to validate the model predictions for a variety of geometric configurations for both the conduction shape factor as well as the full natural convection model. 
Table 1 Enclosure Dimensions for Model Validation

\begin{tabular}{|c|c|c|c|}
\hline \multirow[b]{2}{*}{$L_{o} / L_{i}$} & \multicolumn{3}{|c|}{$\overline{L L / W}$} \\
\hline & 2 & 1 & 0.5 \\
\hline 1.05 & & $\times, \star$ & \\
\hline 1.2 & $\times, \star$ & $\times, \star$ & $\times$ \\
\hline 1.6 & $\times, \star$ & $\times, \star$ & $\times$ \\
\hline 2.0 & & $\times, \star$ & \\
\hline $\begin{array}{l}x-\mathrm{co} \\
\star-\mathrm{cor}\end{array}$ & $\begin{array}{l}\text { etio } \\
\text { ctior }\end{array}$ & & \\
\hline
\end{tabular}

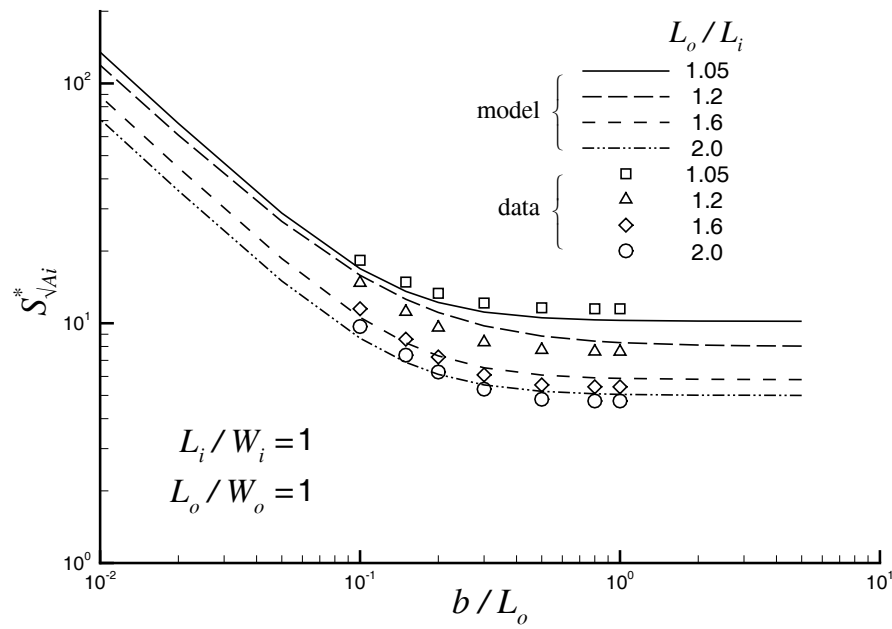

Fig. 7 Conduction Shape Factor Model Validation: $L / W=1$

\section{Conduction Shape Factor}

The conduction shape factor model is validated using CFD data from Flotherm [13] conduction-only simulations for a range of enclosure geometry configurations. Based on a fixed value for the length of the vertical plate, $L_{i}=0.1 \mathrm{~m}$, the remaining dimensions for each enclosure are expressed based on the dimensionless parameters from Eq. (1). Cross-sectional aspect ratios, $L_{o} / W_{o}$ and $L_{i} / W_{i}$, as well as relative inner to outer dimensions, $L_{o} / L_{i}$, included in the validation study are summarized in Table 1. Dimensionless gap spacing values for the $L_{o} / W_{o}=1$ cases were:

$$
b / L_{o}=0.1,0.15,0.2,0.3,0.5,0.8,1.0
$$

while for $L_{o} / W_{o}=2$ the gap spacing values used for the model validation were:

$$
b / L_{o}=0.025,0.05,0.075,0.1,0.15,0.25,0.4,0.5
$$

Based on the comparison with the numerical conduction data for all enclosure configurations, a value for the combination parameter, $m=3 / 2$, in Eq. (16) is selected that minimizes the RMS \% difference for all cases.

A validation of the model for the conduction shape factor with the numerical data is presented in Figs. 7 and 8 for $L_{o} / W_{o}=1$ and 2, respectively. Both of these graphs demonstrate the good agreement between the analytical model for the conduction shape factor and the numerical data for the full range of geometric parameters. Table 2 summarizes the RMS \% difference between the data and the model for all cases examined in this work. The agreement between the model and data is best
Table 2 Model Validation Results: Conduction Shape Factor

\begin{tabular}{|c|c|c|}
\hline \hline$L_{o} / L_{i}$ & $L / W$ & RMS \% \\
\hline 1.05 & 1 & 9.0 \\
1.2 & 1 & 12.8 \\
1.6 & 1 & 7.4 \\
2.0 & 1 & 6.9 \\
1.2 & 2 & 5.3 \\
1.6 & 2 & 6.0 \\
\hline \hline
\end{tabular}

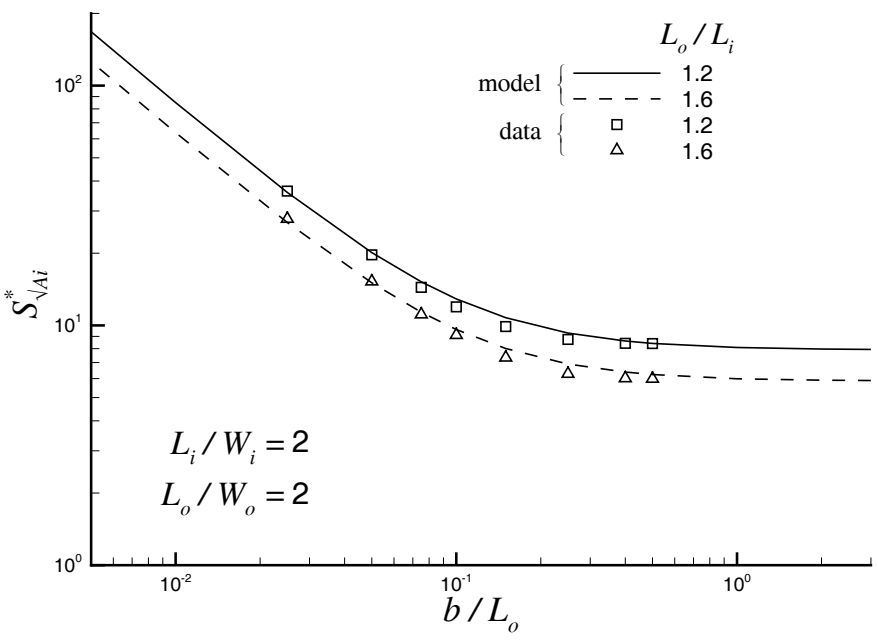

Fig. 8 Conduction Shape Factor Model Validation: $L / W=2$

in the case of larger values of $L_{o} / L_{i}$, while at small $L_{o} / L_{i}$ values the approximation of the enclosure as an equivalent spherical shell is not as effective.

\section{Natural Convection}

The model for natural convection in the enclosure is validated using data from the Icepak [14] CFD simulations described in the previous section. The length of the inner plate in the direction of the gravity vector, $L_{i}=0.1 \mathrm{~m}$ was fixed for all test cases, while the remaining dimensions for the enclosure are presented in Table 1 and also include:

$$
b / L_{o}=0.05,0.1,0.2,0.3,0.5,0.8,1.0
$$

for all values of $L / W$.

From a comparison of the data and model for all test cases examined in this work, a value for the combination parameter in Eq. (6) was selected, $n=1$, that provides the lowest combined RMS \% difference. The resulting general form of the model is:

$$
N u_{\sqrt{A_{i}}}=S_{\sqrt{A_{i}}}+\frac{1}{1 / N u_{\mathrm{bl}}+1 / N u_{\mathrm{tr}}}
$$

where $S_{\sqrt{A_{i}}}^{\star}$ is determined based on Eq. (16) and $N u_{\mathrm{bl}}$ and $N u_{\mathrm{tr}}$ are calculated from Eqs. (17) and (27), respectively.

The model is compared with the data for four different $L_{o} / L_{i}$ configurations where $L_{o} / W_{o}=L_{i} / W_{i}=1$ in Figs. 9 - 12, while the RMS \% difference is presented for each $b / L_{o}$ case in Table 3. The agreement between the model and data is quite good, given the level of the approximations used during the development of the model, with an RMS \% difference over most cases 
Table 3 RMS \% Difference Between Natural Convection Model and Data: $L / W=1$

\begin{tabular}{|c|c|c|c|c|c|}
\hline \hline & \multicolumn{5}{|c|}{$b / L_{o}$} \\
\hline$L_{o} / L_{i}$ & 1.0 & 0.5 & 0.2 & 0.1 & 0.05 \\
\hline 1.05 & 10.8 & 10.4 & 8.0 & 3.5 & 2.6 \\
1.2 & 5.8 & 6.2 & 9.2 & 3.4 & 4.4 \\
1.6 & 10.8 & 6.9 & 7.0 & 5.6 & 9.2 \\
2.0 & 6.2 & 7.0 & 7.1 & 7.0 & 20.7 \\
\hline \hline
\end{tabular}

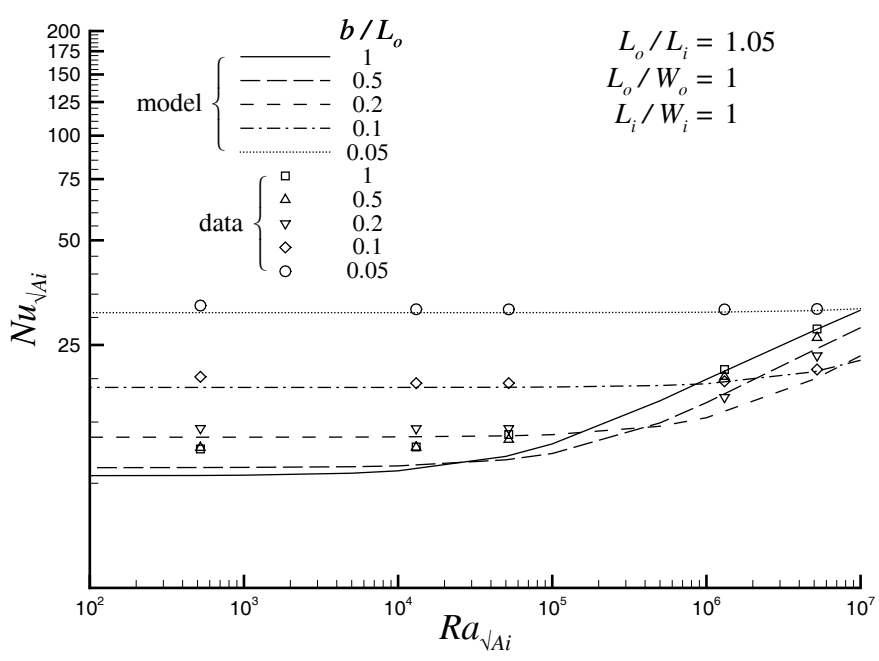

Fig. 9 Convection Model Validation: $L / W=1, L_{o} / L_{i}=1.05$

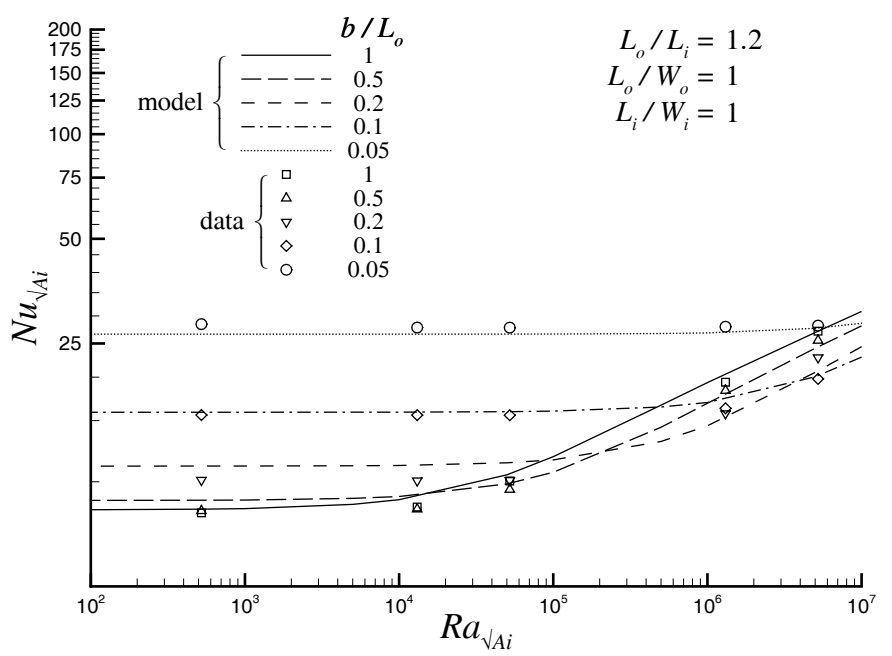

Fig. 10 Convection Model Validation: $L / W=1, L_{o} / L_{i}=1.2$

of less than $10 \%$. Figures $9-12$ also demonstrate the importance of the conductive limit in all the the enclosure geometries examined in this work, which contributes from approximately $50 \%$ to almost $100 \%$ of the total heat transfer, depending on the gap thickness and relative size of the enclosure. The trends of the solution, including the transition from conduction to convection-dominated heat transfer, are all clearly reflected in the numerical data as well.

For enclosure configurations where the cross-sectional aspect ratio is $L_{o} / W_{o}=0.5$ and 2.0, the model is compared with numerical data for two different $L_{o} / L_{i}$ configurations, $L_{o} / L_{i}=$
Table 4 RMS \% Difference Between Natural Convection Model and Data: $L / W=0.5,2.0$

\begin{tabular}{|c|c|c|c|c|c|c|}
\hline \hline & & \multicolumn{5}{|c|}{$b / L_{o}$} \\
\cline { 3 - 7 }$L / W$ & $L_{o} / L_{i}$ & 1.0 & 0.5 & 0.2 & 0.1 & 0.05 \\
\hline 0.5 & 1.2 & 7.2 & 6.8 & 5.0 & 3.8 & 6.5 \\
0.5 & 1.6 & 6.4 & 6.8 & 6.4 & 4.6 & 8.2 \\
2.0 & 1.2 & 16.5 & 17.1 & 14.6 & 11.4 & 12.1 \\
2.0 & 1.6 & 7.5 & 9.0 & 7.9 & 7.1 & 14.4 \\
\hline \hline
\end{tabular}

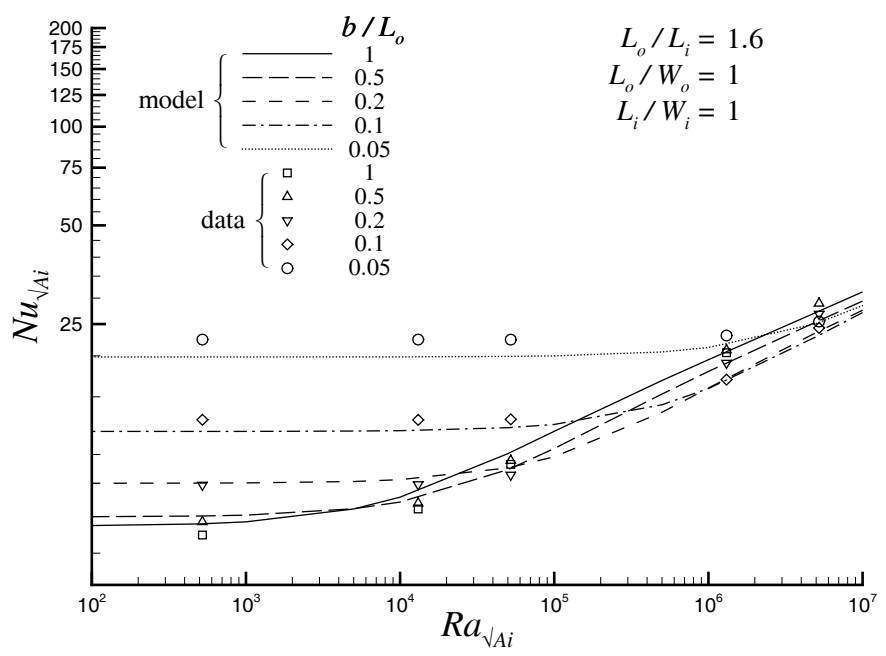

Fig. 11 Convection Model Validation: $L / W=1, L_{o} / L_{i}=1.6$

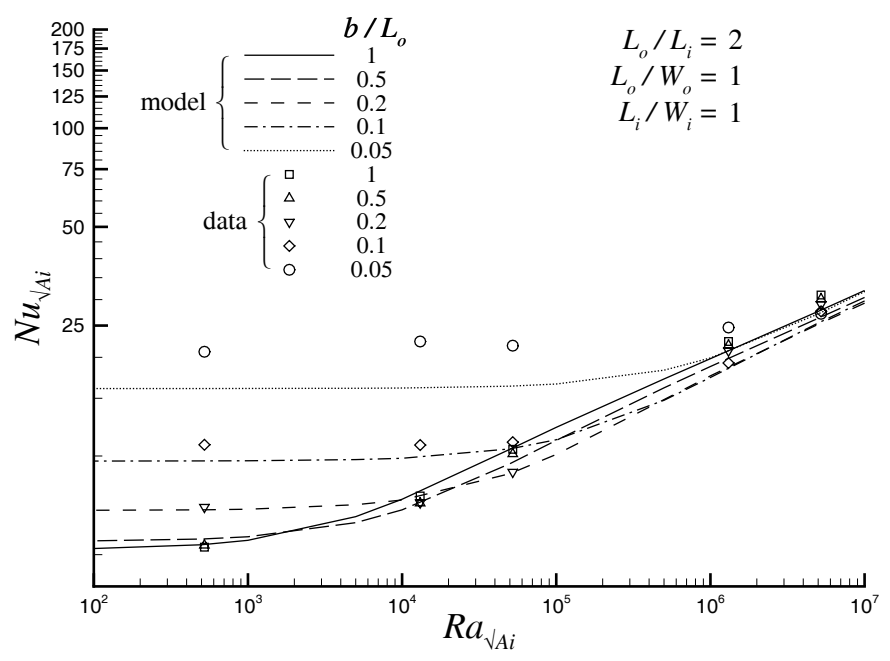

Fig. 12 Convection Model Validation: $L / W=1, L_{o} / L_{i}=2.0$

1.2 and 1.6, as presented in Figs. 13 - 16. The RMS \% difference between the data and the model predictions are given in Table 4.

As in the previous cases, the agreement between the model and the data is quite good, with an average RMS difference of less than $10 \%$ over the full range of enclosure geometries except for the $L / W=2, L_{o} / L_{i}=1.2$ case where the differences are larger. In general, the model and the data agree for most configurations to within the uncertainty in the numerical solution. However, some of the simplifications used in the development of the model, in particular the conduction shape factor, result in 


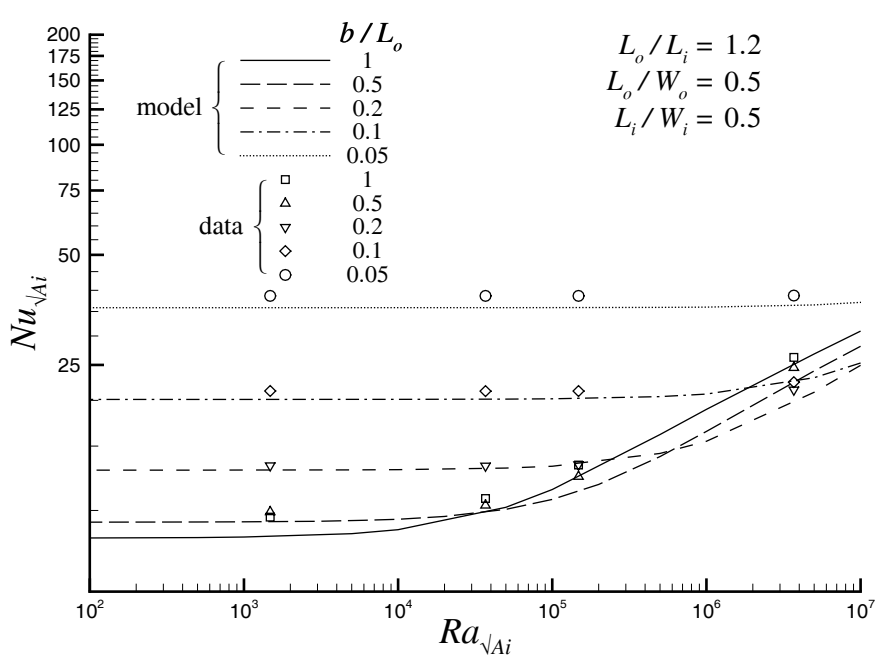

Fig. 12 Convection Model Validation: $L / W=0.5, L_{o} / L_{i}=1.2$

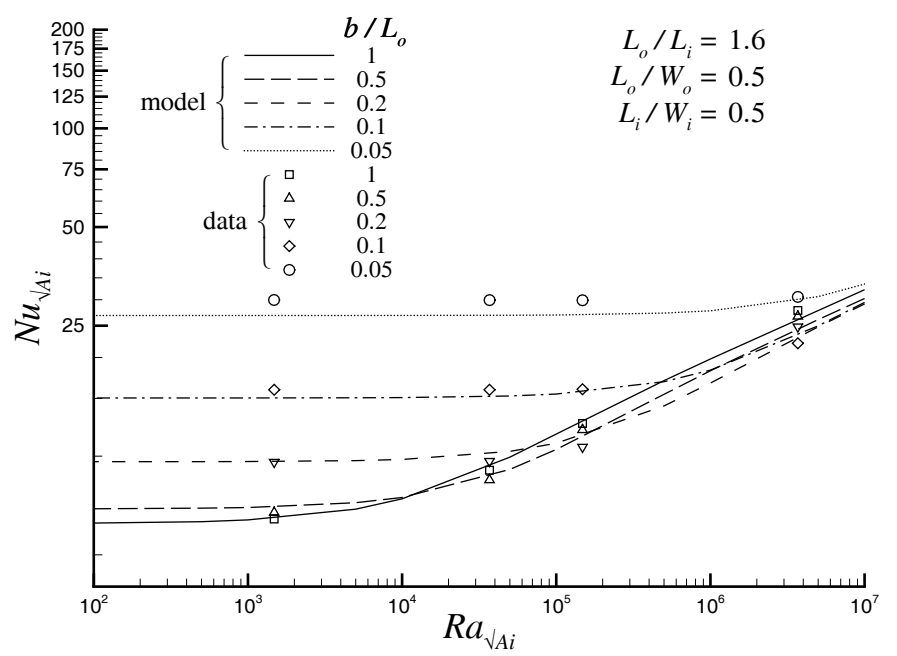

Fig. 13 Convection Model Validation: $L / W=0.5, L_{o} / L_{i}=1.6$

larger differences between the data and the model, especially at certain limiting cases such as small $b / L_{o}$ or large $L_{o} / L_{i}$ values.

\section{SUMMARY AND CONCLUSIONS}

An analytically-based model has been developed that predicts the total heat transfer rate for natural convection from a vertical, isothermal plate to its surrounding isothermal cuboid-shaped enclosure. The model is comprised of three asymptotic solutions, corresponding to pure conduction through the enclosed region, as well as laminar boundary layer flow and transition flow convection. By combining these three independent solutions into a single, composite expression, the resulting model is valid over a wide range of both enclosure / plate geometries and flow conditions. By its nature, the composite model correctly treats limiting cases: the conductive limit for small gap spacing, $b / L_{o}$, and Rayleigh number; and the laminar boundary layer flow limit, achieved at large enclosure dimensions when the behaviour of the vertical plate approaches that of an isolated body in a full space domain. The model requires no correlating coefficients and is not limited to a certain range of values of the

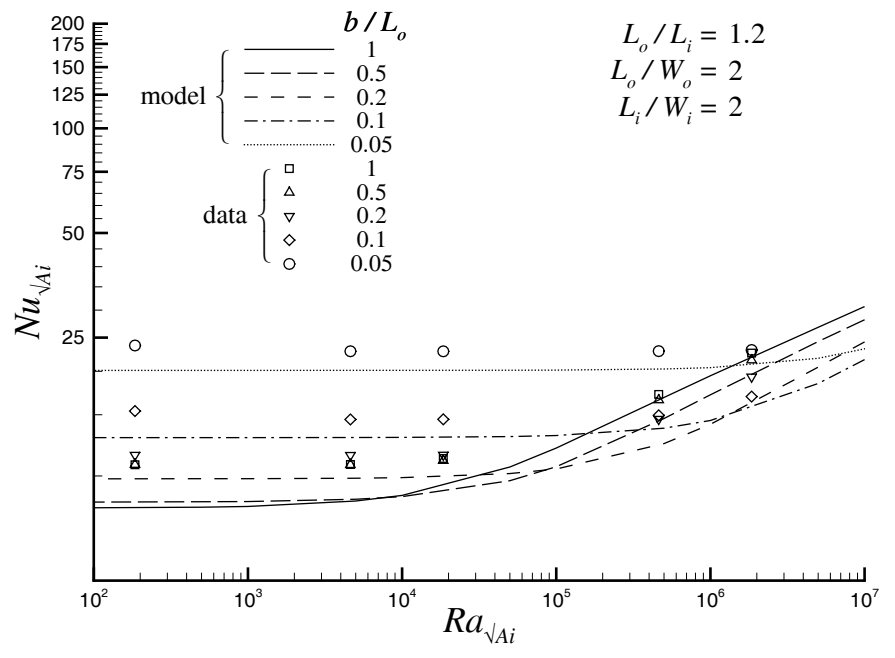

Fig. 14 Convection Model Validation: $L / W=2, L_{o} / L_{i}=1.2$

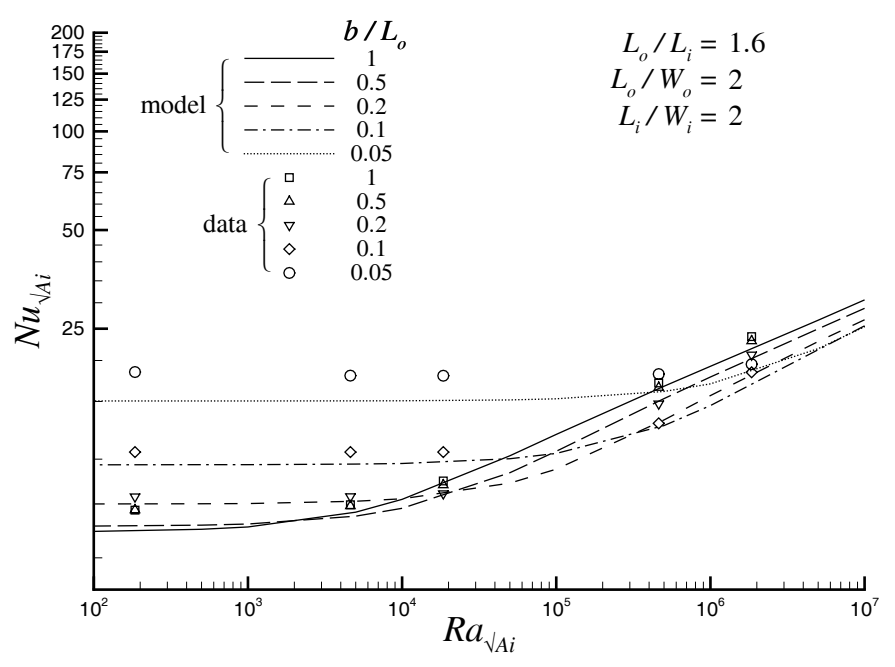

Fig. 15 Convection Model Validation: $L / W=2, L_{o} / L_{i}=1.6$

independent parameters, as is often the case with typical correlations of numerical or experimental data.

The models developed in this study were validated using numerical data from two different commercial CFD software packages and agreement between the models and the data was within an average of $10 \%$ for most enclosure configurations. It should be noted that the numerical results were not validated other than in a grid convergence study, and that these data will almost certainly have a level of error associated with them as well. This may account for the larger differences noted between the data and the model for certain combinations of enclosure dimensions. The model is also effective at demonstrating trends in the data as a function of geometry and Rayleigh number, particularly the importance of conduction to the overall heat transfer rate for small $R a_{\sqrt{A_{i}}}$ and / or narrow gap spacing, $b / L_{o}$.

The model developed in this paper provides an effective method for the sizing of enclosures, the planning of system or circuit board cooling for sealed equipment enclosures, and for performing order-of-magnitude and similar parametric studies. Although not a replacement for CFD analysis or prototype testing, the proposed model provides a quick and easy-to-use al- 
ternative that can be easily programmed into a spreadsheet or symbolic mathematical environment and used during the preliminary design process.

Research and analysis on the topic of natural convection in electronics enclosures will continue with an extension of the current model to include a uniform heat flux boundary condition on the plate, as well as an array of vertical parallel plates in the interior of the enclosure. An experimental test program is also being considered to further validate the models developed in this study.

\section{ACKNOWLEDGMENTS}

The authors gratefully acknowledge the Centre for Microelectronics Assembly and Packaging and Materials and Manufacturing Ontario for their continued support of this work.

\section{REFERENCES}

[1] Park, S.K., "Natural Convection Air Cooling between Vertical Parallel Plates with Heated Protrusions Simulating PlateMounted Electronic Components within an Enclosure," EEP Vol. 26-1, Advances in Electronic Packaging, American Society of Mechanical Engineers, New York, pp. 501 - 509, 1999.

[2] Tang, L. and Joshi, Y.K., "Integrated Thermal Analysis of Natural Convection Air Cooled Electronic Enclosure," ASME Journal of Electronic Packaging, Vol. 121, pp. 108 - 115, 1999.

[3] Symons, J.G., Mahoney, K.J. and Bostock, T.C., "Natural Convection in Enclosures with Through-Flow Heat Sources," Proceedings of the 1987 ASME/JSME Thermal Engineering Joint Conference, Vol. 2, pp. 215 - 220, 1987.

[4] Yang, M. and Tao, W.Q., “Three-Dimensional Natural Convection in an Enclosure with an Internal Isolated Vertical Plate," ASME Journal of Heat Transfer, Vol. 117, pp. 619 - 625, 1995.

[5] Teertstra, P.M., Models and Experiments for Laminar Natural Convection from Heated Bodies in Enclosures, Ph.D. Thesis, Department of Mechanical Engineering, University of Waterloo, Waterloo, Ontario, Canada, 2003.
[6] Teertstra, P.M., Yovanovich, M.M. and Culham, J.R., "Analytical Modelling of Natural Convection in Concentric Spherical Enclosures," 42nd Aerospace Sciences Meeting and Exhibit Conference, Reno, NV, Jan. 5 - 7, 2004.

[7] Churchill, S.W. and Usagi, R., "A General Expression for the Correlation of Rates of Transfer and Other Phenomenon," A.I.Ch.E. Journal, Vol. 18, pp. 1121 - 1128, 1972.

[8] Yovanovich, M.M., "Conduction and Thermal Contact Resistances (Conductances)," Handbook of Heat Transfer, 3rd. ed., eds. W. M. Rohsenow, J. P. Harnett and Y. Cho, McGraw Hill, New York, Chapter 3, pp. 3.1 - 3.73, 1998.

[9] Churchill, S.W. and Churchill, R.U., "A Comprehensive Correlating Equation for Heat and Component Heat Transfer by Free Convection," American Institute of Chemical Engineers Journal, Vol. 21, pp. 604 - 606, 1975.

[10] Yovanovich, M.M. and Jafarpur, K., "Models of Laminar Natural Convection from Vertical and Horizontal Isothermal Cuboids for all Prandtl Numbers and All Rayleigh Numbers Below 1011," Fundamentals of Natural Convection, ASME HTD Vol. 264, pp. 111 - 126, 1993.

[11] Lee, S., Yovanovich, M. M. and Jafarpur, K., "Effects of Geometry and Orientation on Laminar Natural Convection Heat Transfer from Isothermal Bodies," Journal of Thermophysics and Heat Transfer, Vol. 5, pp. 208 - 216, 1991.

[12] Batchelor, G.K., "Heat Transfer by Free Convection Across a Closed Cavity Between Vertical Boundaries at Different Temperatures," Quarterly of Applied Mathematics, Vol. 12, No. 3, pp. 209 - 223, 1954.

[13] Flomerics Inc., 257 Turnpike Rd. Suite 100, Southborough, MA, 2004.

[14] Fluent Inc., 10 Cavendish Court, Lebanon, NH, 2004. 